\title{
Femtosecond studies of electrons in liquids
}

\author{
Hong Lu, Frederick H. Long, and K. B. Eisenthal \\ Department of Chemistry, Havemeyer Hall, Columbia University, New York, New York 10027
}

Received February 8, 1990; accepted April 3, 1990

\begin{abstract}
Femtosecond time-resolved measurements were performed on the geminate recombination and solvation of electrons in liquids. The electrons were generated by multiphoton ionization in neat water and alkanes and by multiphoton detachment from $\mathrm{Cl}^{-}$and $\mathrm{OH}^{-}$ions in aqueous solution. For the neat liquids, the results are compared with the Onsager model of ion-pair recombination. An isotope effect is observed in the dynamics of both solvation and recombination in neat $\mathrm{D}_{2} \mathrm{O}$ relative to $\mathrm{H}_{2} \mathrm{O}$. Studies with improved time resolution of electron solvation in neat water have measured the formation, decay, and absorption spectrum of the wet electron, i.e., the presolvated electron. By the discovery of an isosbestic wavelength, the formation of the wet electron is shown to involve two states; i.e., the transition is not continuous. The picture of the wet electron as an excited state of the solvated electron is presented. The differences in the solvation dynamics of electrons that are generated from neat water and from $\mathrm{OH}^{-}$and $\mathrm{Cl}^{-}$ions show that short-range molecular effects are important.
\end{abstract}

\section{INTRODUCTION}

The properties of electrons in liquids have been of great interest to a large community of researchers since the discovery of electrons in liquid ammonia more than 100 years ago by Weyl. ${ }^{1-7}$ In particular, electrons in aqueous solution, i.e., solvated electrons, have long ago been recognized to be relevant to a wide variety of physical problems, such as electrons in amorphous solids, ${ }^{7}$ and biological problems, such as electron transfer. ${ }^{8}$ Clearly, a better understanding of the solvated electron will greatly advance the understanding at a microscropic level of many chemical, physical, and biological processes.

Previously, high-energy electron beams were used to study electrons in liquids. ${ }^{3-6}$ However, this method suffers from several significant drawbacks. First, owing to the highly energetic electrons used, it was impossible to ensure that secondary reactions were not masking the dynamics of interest. Often multiple electron-cation pairs in close proximity (spurs) were produced, thereby making it difficult to interpret the data. Second, these studies did not have sufficient time resolution to study fast processes such as electron solvation or electron-cation recombination. Femtosecond spectroscopy solves both these fundamental problems. In addition to permitting time resolution of many of the fundamental processes that involve the interaction of an electron with a solvent, with the peak powers that are typically available from an amplified colliding-pulse mode-locked laser $\left(I \approx 10^{13} \mathrm{~W} / \mathrm{cm}^{2}\right)$ femtosecond spectroscopy allows only single electroncation pairs to be produced.

In previous publications we discussed studies of electron-cation recombination in neat alkanes and neat water and electron-atom geminate recombination in aqueous ionic solution. ${ }^{9-12}$ In this paper we will present new data on electron-cation recombination in a series of alkanes. These results and our measurements in $\mathrm{H}_{2} \mathrm{O}$ and $\mathrm{D}_{2} \mathrm{O}$ will be compared with the Onsager model of geminate electron-cation recombination. Because of their improved time resolution, these studies have made it possible to test more definitively whether classical diffusion can be used to describe electron-cation recombination in neat liquids. ${ }^{13}$

Experiments with electron solvation in neat water and in electrolyte solutions will be presented. Studies with improved time resolution have measured the formation, decay, and absorption spectra of the wet electron. ${ }^{14}$ The discovery of an isosbestic point, i.e., a wavelength at which the absorption is constant as the solvated electron appears, shows for the first time to our knowledge that the wet-tosolvated electron transition involves only two states. In other words, the transition is not continuous. This is fundamentally inconsistent with the dielectric-relaxation picture of electron solvation. The picture of the wet electron as an excited state of the solvated electron is presented. Electrons that are photodetached from simple ions exhibit quite different solvation and recombination dynamics than electrons that are photoionized from neat water. Strong evidence is found for molecular effects on the solvation and recombination dynamics of an electron that is photodetached from a simple ion.

\section{EXPERIMENTS}

The experiments reported here were all performed by using an amplified colliding-pulse mode-locked dye laser that generated a $10-\mathrm{Hz}$ train of pulses $(625-\mathrm{nm}, 300-$ $500 \mu \mathrm{J} /$ pulse, full width at half-maximum, 80-100 fsec). A diagram of the pump-probe scheme that was used in these experiments is shown in Fig. 1. The pump beam was formed by frequency doubling the colliding-pulse mode-locked beam with a $200-\mu \mathrm{m} \beta$-barium borate (BBO) crystal. The pump-probe apparatus that was used is shown in Fig. 2. The probe beam either was a small fraction of the remaining fundamental or was part of a whitelight continuum that was created by focusing the fundamental beam into a 1-cm path-length $\mathrm{D}_{2} \mathrm{O}$ cell. A usable continuum was created from 400 to $1300 \mathrm{~nm}$. Different wavelengths were selected by using the appropriate broad- 


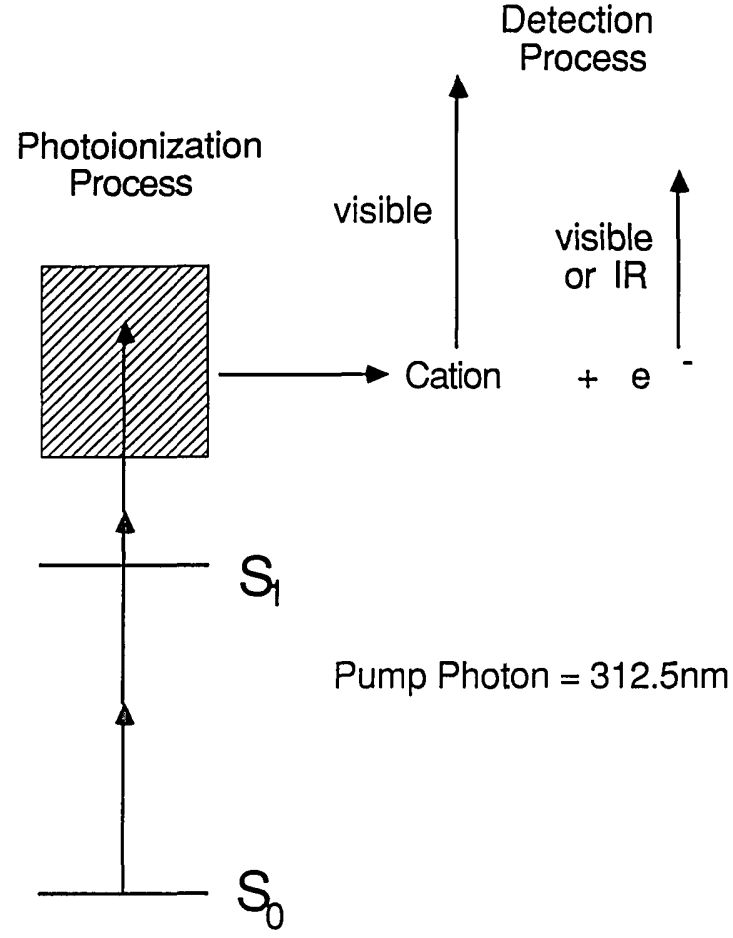

Fig. 1. Schematic diagram of pump-probe scheme used for experiments discussed in this paper. A femtosecond UV-pulse three-photon ionizes a solvent molecule. Either the cation or electron produced can be monitored in time by measuring the transient absorption in the visible or the IR.

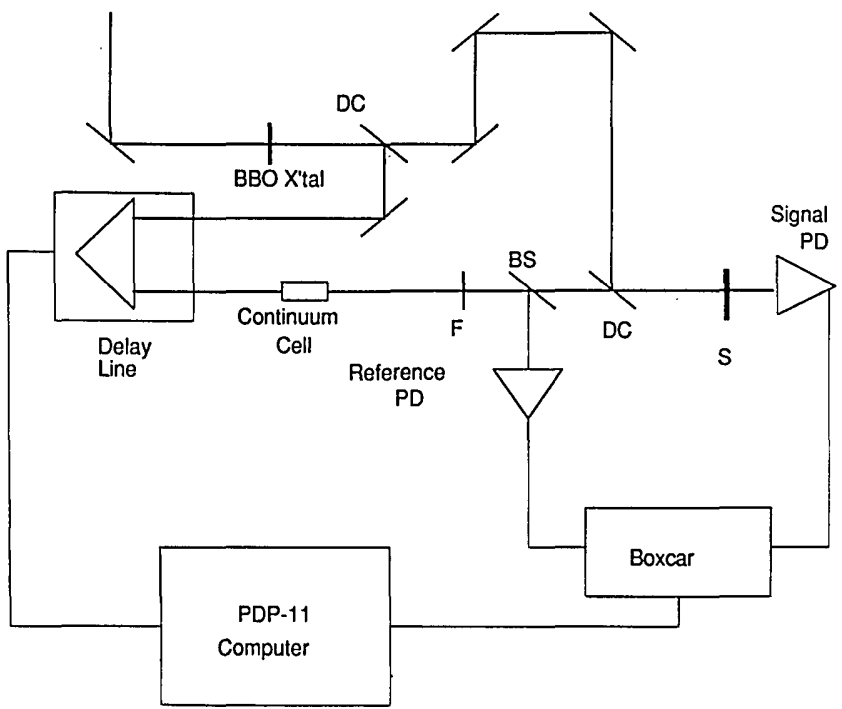

Fig. 2. Schematic diagram of experimental apparatus. DC's, dichroic beam splitters; BS, beam splitters; PD's, photodiodes; F, interference filter; $\mathrm{S}$, sample.

band (10-nm full width at half-maximum) interference filter. The recorded signal was the difference of the probe beam and a reference, divided by the reference. Typically, changes in transmission of the order of $10^{-3}$ can be measured.

The neat alkanes were purified by column distillation before use. The $\mathrm{H}_{2} \mathrm{O}$ was high-pressure liquid-chromatog- raphy grade, the $\mathrm{D}_{2} \mathrm{O}$ was $99.95 \%$ isotope purity, and the salts were from Aldrich and were used as received.

\section{THEORETICAL BACKGROUND}

This section is divided into two parts: Subsection 3.A is a discussion of the theory of solvation dynamics. The dielectric-continuum model (DCM) and more sophisticated theoretical approaches are discussed. Subsection 3.B is a discussion of geminate-pair recombination for both ion pairs, the so-called Onsager theory, and neutral-pair recombination for which no attractive force is present.

\section{A. Electron Solvation Dynamics}

One of the early theories of solvation is the DCM. ${ }^{15-17}$ In this model the solvent that surrounds the electron or dipole is treated as a continuum dielectric with a frequencydependent dielectric constant $\varepsilon(\omega)$. The dielectric response times are given by the transverse time $\tau_{D}$ and the longitudinal time $\tau_{L}$ by $\tau_{D}\left(\varepsilon_{\infty} / \varepsilon_{0}\right)$. In more recent years there have been a number of more sophisticated treatments and applications of the DCM of electron solvation. ${ }^{18-23}$ Analytic molecular theories that go beyond the predictions of the DCM are scarce, the most notable being the mean-spherical-approximation model that was originated by Wolynes and extended by other researchers. ${ }^{24-26}$ Because of the complexity of the interactions of an electron with a polar-fluid-like water, it would be surprising if analytic theories alone could completely solve this problem. Perhaps the best hope for a theoretical understanding of solvation dynamics comes from computer simulations. Recent studies that used path-integral Monte Carlo techniques have included attempts to calculate the equilibrium solvated-electron absorption spectra in water. ${ }^{27,28}$ There have already been quantum-mechanical simulations of solvation dynamics in water. ${ }^{29,30}$ Most relevant to our research are the studies of Rossky and Schnitker, who have predicted time-dependent spectra for the electron. ${ }^{29}$ In addition, isotope effects on the solvation dynamics of an electron in water were predicted recently by Barnett et al..$^{30}$ A detailed comparison of our solvation data with the results of simulations is in progress.

\section{B. Electron-Cation Geminate Recombination}

It was realized long ago by Onsager that the motion of thermalized electrons in some liquids could be described as diffusional. ${ }^{31}$ This theory, the Onsager model, assumes that electron-recombination kinetics can be described by a Brownian particle in the presence of a Coulombic field; see Fig. 3. Below we will briefly discuss the assumptions that underlie this theory. To begin, we require an equation for the electron probability density, $\rho(r, t)$. We can use conservation of probability,

$$
\frac{\partial \rho(\mathbf{r}, t)}{\partial t}+\operatorname{div} \mathbf{j}=0,
$$

to relate $\rho(r, t)$ to $j$, the probability current, which can be written as the sum of two terms, the Fick's law or freediffusion term and the drift term; drift is caused by the external electric field of the cation:

$$
\mathbf{j}=-D \nabla \rho+\mu \mathbf{E}_{\text {ext }} \rho,
$$




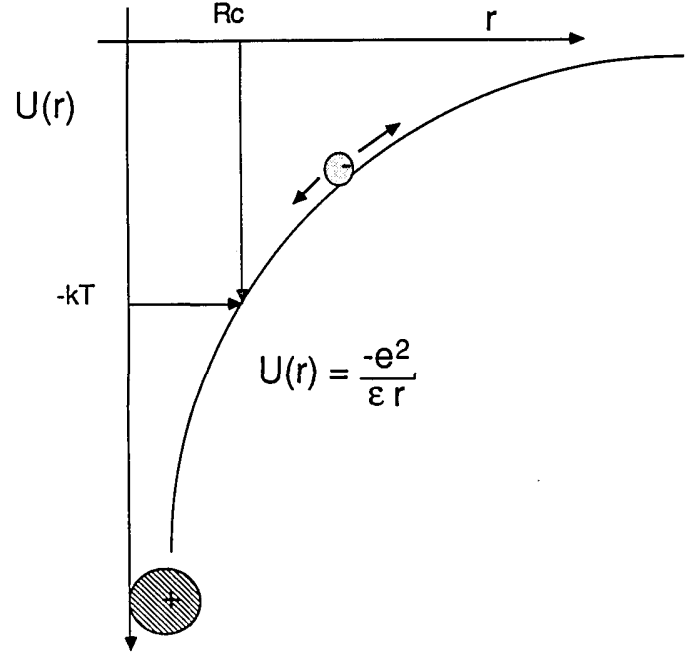

Fig. 3. Illustration of Onsager model of geminate electroncation recombination. The motion of the electron is assumed to be a Brownian particle inside a Coulombic force field. The relevant length scale is the Onsager radius, i.e., the distance at which the potential energy is equal to $k T$.

$$
\mathbf{E}_{\mathrm{ext}}=\frac{q_{\mathrm{ion}} \hat{r}}{\varepsilon r^{2}}
$$

where $e$ is the elementary charge, $\varepsilon$ is the dielectric constant, $D$ is the diffusion coefficient, and $\mu$ is the electron mobility, i.e., the ratio of the drift velocity to the applied electric field. The electron-diffusion coefficient is related to the low-field electron mobility, which can be measured experimentally, by an Einstein relation

$$
D=\frac{k T}{e} \mu
$$

This equation is often put into the following form:

$$
\frac{\partial \rho}{\partial t}=D \operatorname{div}\left\{\exp \left(r_{c} / r\right) \nabla\left[\exp \left(-r_{c} / r\right)\right]\right\},
$$

where $r_{c}$ is the so-called Onsager radius, which is

$$
r_{c}=\frac{e^{2}}{\varepsilon k T}
$$

The Onsager radius is the distance at which the potential energy of the electron-cation pair is equal to the thermal energy $k T$. For a typical alkane at room temperature, $r_{c}$ is $\sim 300 \AA$. This does not vary by more than $5 \%$ for the alkanes that are considered in this study. However, for water the Onsager length is $\sim 7 \AA$, if the static dielectric constant is used. In an experiment one measures not $\rho\left(r, r^{\prime}, t\right)$, the probability density that the electron at $r$ at $t=0$ is at $r^{\prime}$ at time $t$, but the time-dependent survival probability, $\Omega(r, t)$. $\Omega$ is simply related to $\rho\left(r, r^{\prime}, t\right)$ by Eq. (7):

$$
\Omega(r, t)=\lim _{a \rightarrow 0} \int_{a}^{\infty} \mathrm{d} r^{\prime} r^{\prime 2} 4 \pi \rho\left(r, r^{\prime}, t\right) .
$$

Physically, $\Omega(r, t)$ is the probability of the electron's escaping recombination with the parent cation, given that the electron starts out thermalized at a distance $r$ from the cation at $t=0$. Onsager was the first person to study this problem quantitatively; he found that the asymptotic value of $\Omega,(t \rightarrow \infty)$ was given by the simple result

$$
\Omega(r)=\exp \left(-r_{c} / r\right) .
$$

Simple derivations of this result and generalizations of it have been done by Tachiaya. ${ }^{32}$ Several others have worked on approximate solutions to the more general time-dependent problem. ${ }^{33,34}$ Hong and Noolandi have developed an exact analytic solution to the problem. ${ }^{35}$ Unfortunately, their solution is quite complex; however, they have numerically solved for $\Omega(r, t)$ by using their analytic results. ${ }^{6,35}$

Neutral-pair recombination has been studied by many workers. To the best of our knowledge, the first calculation of the geminate-pair recombination kinetics was done by Collins and Kimball. ${ }^{36}$ The force between the two particles was taken to be zero, so that the finite sizes of the particles had to be included in order for recombination to take place. The result that these authors obtained for the escape probability $\Omega(r, t)$, is relatively simple:

$$
\Omega(R, t)=1-\frac{r_{a}}{R} \operatorname{erfc}\left(\frac{R-r_{a}}{\sqrt{4 D t}}\right),
$$

where $D$ is the mutual diffusion coefficient, $R$ is the initial separation distance of the two particles, and $r_{a}$ is the reaction radius, i.e., the sum of the particle radii. This result also assumes that there is no significant barrier to the reaction, and thus the rate constant is diffusion limited.

There are many implicit assumptions in the above theories. We assume that on the time scale of these measurements the motion of the electron is diffusive. This can be roughly justified by the following argument. From the experimental electron mobility, a friction coefficient and an effective collision time can be found. With the thermal velocity of the electron, a mean free path for the electron can be calculated. In the case of iso-octane this path is $\sim 2 \AA$. Since this length is much shorter than either the Onsager length $(300 \AA)$ or the thermalization distance, the motion can be considered to be diffusive. Recent simulations by Schnitker and Rossky have shown that a solvated electron in water moves diffusionally on the picosecond time scale. ${ }^{37}$ The finite sizes of the cation and the electron are neglected in the Onsager problem. This is not important for the alkane results but is possibly important for electron recombination in water; see Section 4. Furthermore, the possibility of hydrodynamic interactions has not been considered in detail; however, the strength of those interactions would be expected to be weak compared with that of the Coulombic interaction. ${ }^{38}$

\section{RESULTS AND DISCUSSION}

\section{A. Electrons in Neat Alkanes}

Early experimental work on electrons in nonpolar liquids involved the measurement of electron mobilities and recombination escape yields. ${ }^{39-41}$ However, as we mentioned in Section 1, these studies could not address many fundamental question concerning electrons in liquids. With the development of amplified picosecond and fem- 


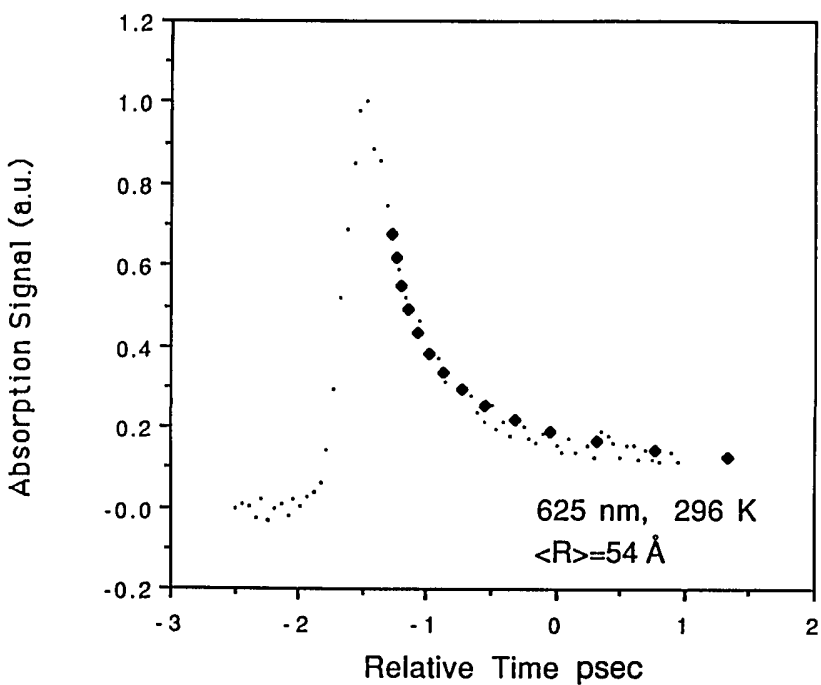

Fig. 4. Electron-cation geminate recombination in iso-octane (2,2,4-trimethylpentane) after UV pump pulse (small dots; $\left.T=296 \mathrm{~K}, \lambda_{\text {probe }}=625 \mathrm{~nm}\right)$. The Hong-Noolandi ${ }^{35}$ prediction is superimposed (large dots), with $\langle r\rangle=54 \AA$ (exponential distribution), $\mu=5.8 \mathrm{~cm}^{2} \mathrm{sec}^{-1} \mathrm{~V}^{-1}, T=296 \mathrm{~K}, \varepsilon=1.94$; see text for further details.

tosecond lasers in recent years, there has been a renewed interest in this problem. Several studies have been done with picosecond laser pulses or electron beams. ${ }^{13,42,43}$ However, these studies did not have sufficient time resolution to resolve the recombination dynamics fully. Femtosecond spectroscopy solves these fundamental experimental problems by improving the time resolution and permitting the photoionization of neat liquids as well as solutes.

In Fig. 4, typical data for iso-octane (2,2,4-trimethylpentane), taken with a $625-\mathrm{nm}$ probe pulse, is shown. The results at 1000 and $625 \mathrm{~nm}$ show the same kinetic behavior as for iso-octane. These measurements indicate that the electron-cation geminate recombination in the nearly spherical, high-electron-mobility solvent (electron low-field drift mobility $\mu=5.8 \mathrm{~cm}^{2} \mathrm{~V}^{-1} \mathrm{sec}^{-1}$; see Table 1) is essentially finished in $\sim 1$ psec. With the solutions to the Onsager model obtained by Hong-Noolandi theory, a more careful analysis of our kinetics can be done. The Hong-Noolandi calculation ${ }^{35}$ describes the geminate recombination kinetics for a single cation-electron pair at a given initial distance of separation. It is physically more reasonable to assume that a distribution of separation distances is formed during the thermalization of the ejected electrons. Following the approach of Scott and Braun, ${ }^{13}$ we integrate over a distribution of electron-cation thermalization distances $g(r)$, where $\Omega(r, t)$ is the electron survival probability as a function of time for a given initial electron-cation separation:

$$
\Omega(t)=\int \mathrm{d}^{3} r g(r) \Omega(r, t) .
$$

We used an exponential distribution of thermalization distances,

$$
g(r)=\frac{1}{8 \pi L^{3}} \exp (-r / L)
$$

It should be noted that a Gaussian distribution gave similar results. A simple calculation shows that $\langle r\rangle$, the mean distance, is $3 L$ for the exponential distribution given in Eq. (11). ${ }^{44}$ A fit was done by varying $L$ and using the experimentally known value for $\mu$. It is known that in high fields the assumption of a linear response is not valid. ${ }^{40}$ However, the kinetics of geminate recombination will be determined chiefly by the length of time that the electron spends far from the cation, where the Coulombic attraction is weaker, rather than by the shorter time that the electron spends near the cation, where the field is high and the assumptions that are inherent in a continuum description of the solvent will break down. Thus we believe that this approach gives a valid first approximation. The above analysis yields a mean electron thermalization distance of $54 \AA$ in iso-octane. We estimate the error in our approximation of $\langle r\rangle$ to be $\pm 20 \%$. The error is relatively large in the alkanes because the final escape yield is only a small fraction of the electrons produced and is therefore just slightly above the signal-to-noise level. As expected, our $r$ is much smaller than the value of $105 \AA$ obtained by Schmidt and Allen, who used electron-beam methods. ${ }^{41}$ This difference was expected; the initial excess energy of the electrons that are produced by electron-beam excitation should be larger because the incident electron is highly energetic. In our experiments the excitation energy is only 3-4 eV greater than the ionization potential.

The electron-cation geminate recombination dynamics in $n$-octane, probed at $1000 \mathrm{~nm}$, is shown in Fig. 5 along with the Hong-Noolandi prediction. In both cases the signal begins to level off after $\sim 30$ psec. A constant back-

\begin{tabular}{|c|c|c|c|c|c|}
\hline Solvent & $\mu_{e}\left(\mathrm{~cm}^{2} \mathrm{~V}^{-1} \mathrm{sec}^{-1}\right)$ & $\rho\left(\mathrm{g} / \mathrm{cm}^{3}\right)$ & I.P. $(\mathrm{eV})_{\mathrm{gas}}{ }^{a}$ & I.P. $(\mathrm{eV})_{\mathrm{liq}}{ }^{a}$ & $\varepsilon$ \\
\hline$n$-Hexane & 0.09 & 0.6603 & 10.18 & 8.7 & 1.885 \\
\hline 2-Methylpentane & 0.29 & 0.6532 & 10.12 & & 1.901 \\
\hline 2,3-Dimethylbutane & 1.1 & 0.6616 & 10.02 & & 1.953 \\
\hline 2,2,4-Trimethylpentane & 7 & 0.6919 & 9.86 & 8.38 & 1.936 \\
\hline 2,2-Dimethylbutane & 12 & 0.6485 & 10.06 & 8.49 & 1.926 \\
\hline
\end{tabular}

Table 1. Solvent Parameters

${ }^{a}$ I.P., ionization potential. 


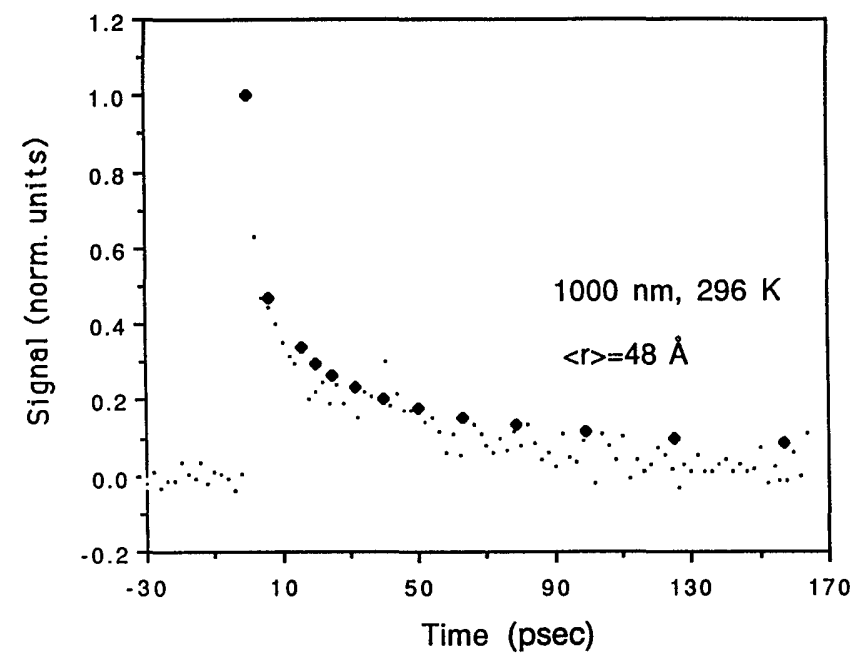

Fig. 5. Electron-cation geminate recombination in octane after UV pump pulse (small dots; $T=296 \mathrm{~K}, \lambda_{\text {probe }}=1000 \mathrm{~nm}$ ). The Hong-Noolandi ${ }^{35}$ prediction (large dots) is superimposed, with $\langle r\rangle=48 \AA$ (exponential distribution), $\mu=0.041 \mathrm{~cm}^{2} / \mathrm{sec}^{-1} \mathrm{~V}^{-1}$, $T=296 \mathrm{~K}, \varepsilon=1.9$; see text for further details.

ground that is caused by excited state absorption has been subtracted. This is consistent with the room-temperature fluorescence lifetime of $n$-octane, $1.4 \mathrm{nsec}^{45}$ Again, it is interesting to compare our data with predictions by the theory of Hong and Noolandi. As before, the mobility used was the experimentally known value for the low-field drift mobility, and the thermalization distance was varied. A mean electron thermalization length of $48 \AA$ was obtained. This result is consistent with that of the studies by Braun and Scott. ${ }^{13}$ They obtained an electron thermalization length of $42 \AA$ for $n$-hexane. However, in their experiments a solute was used, and a different amount of excess energy was assigned to the ejected electron. As expected, these numbers are smaller than the typical thermalization lengths that are obtained by electronbeam excitation for linear alkanes, $60-70 \AA \AA^{41}$ So that we could further examine the kinetics of geminate recombination, the temperature of the sample was varied. These measurements are qualitatively consistent with the diffusion picture and will be discussed in a later publication.

Similar results have been obtained in a series of neat alkanes. Representative results are shown in Fig. 6 for $n$-hexane and 2,3-methylbutane (isohexane). The same qualitative trends that were found in octane and isooctane were found to be true in general. The geminate recombination was found to be faster in spherical, highmobility hydrocarbons. Similar results were obtained when the Onsager model predictions were compared with our measurements and will be discussed in more detail in a later publication.

\section{B. Electrons in Neat Water}

\section{Solvation Dynamics}

Electron-solvation dynamics in water has been of great interest for many years. ${ }^{46-48}$ Electron pulse radiolysis studies showed that the aqueous electron formed very quickly, i.e., in less than a few picoseconds. Early subpicosecond studies in aqueous ferrocyanide solution by
Wiesenfeld and Ippen showed that electron solvation occurs in $\sim 0.3$ psec. ${ }^{49}$ Studies by Migus et al. followed the dynamics of an electron in neat water. ${ }^{50}$ The wet electron was found to undergo a transition to the fully solvated electron in a time approximately equal to the longitudinal dielectric relaxation time in water. Migus et al. claimed that their experimental measurements supported the existence of only two states, the wet and the solvated electron; however, this important point could not be established from their data, since both the wet and the solvated electron absorb in the IR and in the visible regions that were probed. ${ }^{50}$ In order to clarify the situation, we have done experiments with better time resolution and have found strong experimental evidence for the two-state picture by demonstrating the existence of an isosbestic point in the dynamics of the wet-to-solvated electron transition. ${ }^{14}$ This establishment of the two-state model for the transition of the wet electron to the solvated electron is fundamentally inconsistent with the dielectric-relaxation picture of electron solvation.
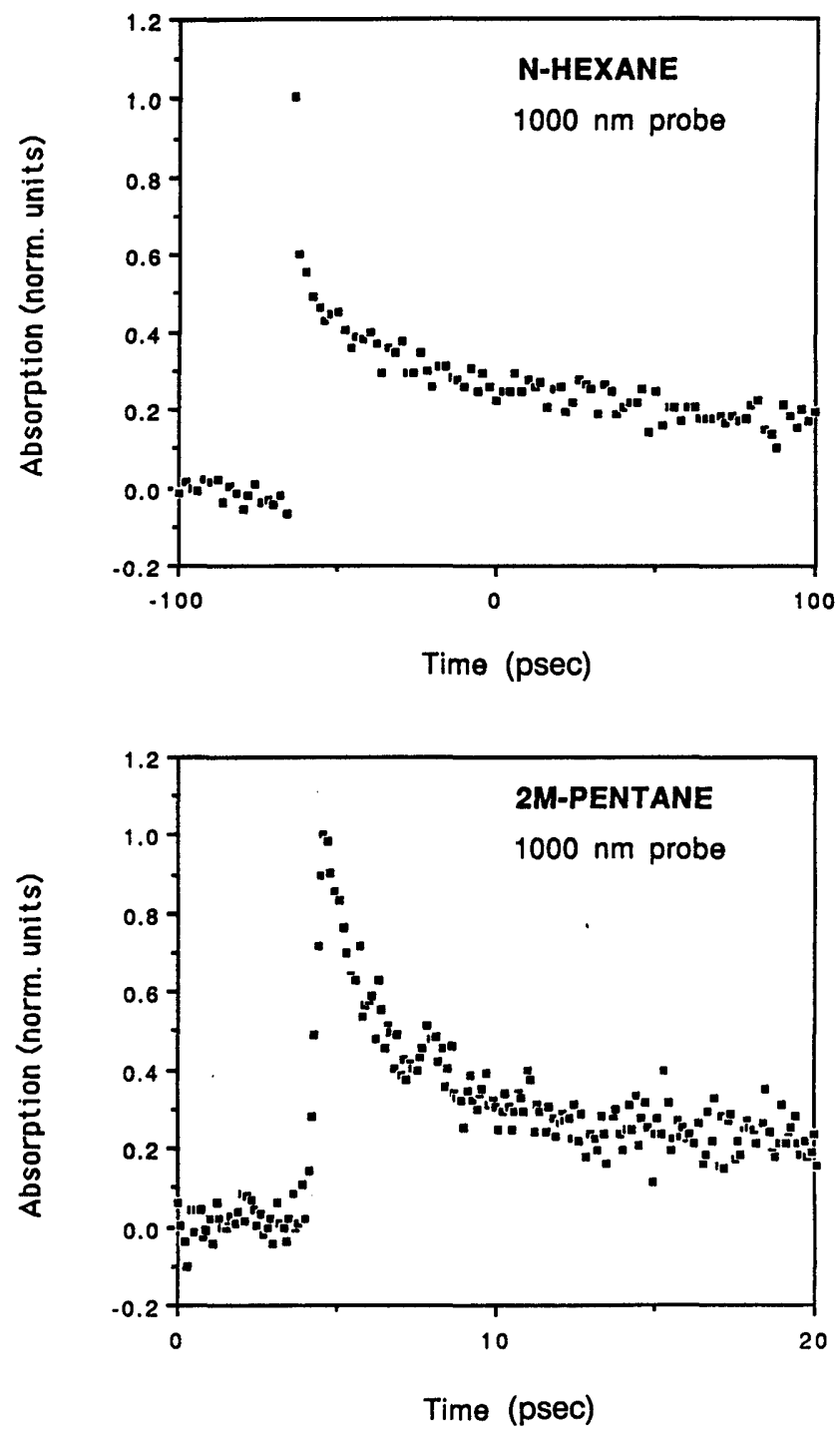

Fig. 6. Electron-cation geminate recombination in $n$-hexane and iso-hexane (2-methyl-pentane) $\left(T=296 \mathrm{~K}, \lambda_{\text {probe }}=625 \mathrm{~nm}\right)$. 


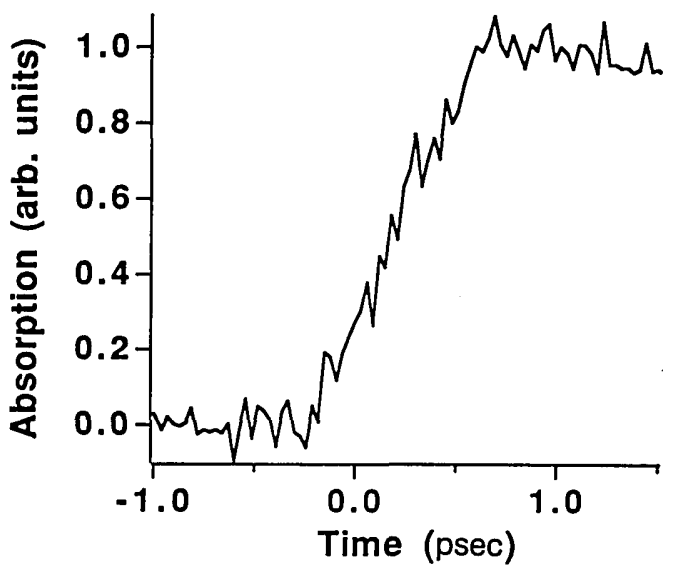

Fig. 7. Time evolution of the aqueous electron, probed at the isosbestic wavelength $(820 \mathrm{~nm})$.

The two-state model implies that a wet electron, which is identifiable by its characteristic absorption spectrum, undergoes a transition to the solvated electron. Thus there would be only two species present in the kinetics rather than a series of wet-electron species that have their own spectra and evolve to the final solvated electron. In the two-state model, if the absorption spectra of the two species (states) overlap, then there must be a wavelength at which the two spectra intersect and therefore have the same absorption coefficient $\varepsilon$. The wavelength of equal absorption is called the isosbestic point. A consequence of the presence of an isosbestic point can be seen by considering the absorption $A(t)$ at a time $t$ and wavelength $\lambda$ :

$$
A(t)=\varepsilon_{\mathrm{wet}} N_{\mathrm{wet}}(t)+\varepsilon_{\mathrm{sol}} N_{\mathrm{sol}}(t),
$$

where $N_{\text {wet }}(t)$ and $N_{\text {sol }}(t)$ are the concentrations of wet and solvated electrons, respectively, at time $t$. At the isosbestic wavelength $\lambda_{l}, \varepsilon_{\text {wet }}=\varepsilon_{\text {sol }}$ and $A(t)=\varepsilon_{\text {wet }}\left[N_{\text {wet }}(t)+N_{\text {sol }}(t)\right]$. The kinetics at $\lambda_{l}$ would therefore be constant after the wet electron is generated, since the total electron concentration $N(t)$ is conserved. At other wavelengths the kinetics should involve a rise or decay, depending on whether the solvated or the wet electron has the larger absorption coefficient. To find the isosbestic point, we scanned from the IR to the visible. At $820 \mathrm{~nm}$ the isosbestic point was found in the formation of the solvated electron from the wet electron, Fig. 7. In other words, the absorption coefficients of the wet and the solvated electron are equal, so that for our experiments the two species are identical, and the transition of the wet electron to the solvated electron is irrelevant. We corrected for a small decay, which is present at times greater than 1 psec owing to the much slower geminate recombination of the solvated electron, by using our previous experimental results. A more detailed discussion of the solvatedelectron geminate recombination is given below, in this section. The existence of an isosbestic point is strong evidence that the wet-to-solvated electron transition involves only two states.

Accordingly, we used a simple kinetic model in order to fit the data:

$$
e_{\text {quasi-free }} \stackrel{k_{1}}{\rightarrow} e_{\text {wet }} \stackrel{k_{2}}{\rightarrow} e_{\text {sol }} .
$$

By solving the rate equations, one obtains

$$
\begin{gathered}
N(t)=N_{0}\left[\frac{k_{1}}{k_{1}-k_{2}}\left(\varepsilon_{\text {wet }}-\varepsilon_{\text {sol }}\right) \exp \left(-k_{2} t\right)+\varepsilon_{\text {sol }}\right. \\
\left.+\left(\frac{k_{2} \varepsilon_{\text {sol }}-k_{1} \varepsilon_{\text {wet }}}{k_{1}-k_{2}}\right) \exp \left(-k_{1} t\right)\right]
\end{gathered}
$$

$N_{0}$ is the number of electrons made by the ionizing pump pulse. Our experimental results show that the wetelectron formation time, $1 / k_{1}$, is $180 \pm 40 \mathrm{fsec}$. At wavelengths other than the isosbestic point, the decay or rise that is due to the wet-electron disappearance causes the fit to be ambiguous and the determination of the rate constant $k_{1}$ to be uncertain. The lifetime of the wet electron, $1 / k_{2}$, was found to be $540 \pm 50 \mathrm{fsec}$. The overall average solvation time of $350 \mathrm{fsec}$ that was found in our early measurements is consistent with our new results; i.e., fitting a two-component rise to a single-exponential rise yields $350 \mathrm{fsec}$ for these measurements. ${ }^{10-12}$ When we use the known values for the solvated-electron absorption, ${ }^{51}$ the wet-electron absorption spectra can be inferred without relying on absolute absorption measurements (Fig. 8). The similarity of the wet-electron absorption to that of the solvated electrons is striking. It can be seen that the wet-electron absorption has a blue tail that extends past $2.5 \mathrm{eV}$; in addition, the absorption has a broad maximum near $900 \mathrm{~nm}$ or $1.4 \mathrm{eV}$, and the overall absorption strength is similar to that of the solvated electron.

We must now consider the physical implication of these results. Our experimental results show that the wet electron forms within $180 \mathrm{fsec}$ after the photoionization of the water molecule. This time is interpreted as the total time that is needed for the electron to localize in a preexisting trap $^{52}$ followed by a further deepening of the well to a configuration that is appropriate to the wet electron. By the discovery of an isosbestic point, the two-state model is verified. This suggests that the wet electron is really an excited state of the solvated electron. This would quite naturally lead to the two states seen in the dynamics. The excited state is likely to be the lowest excited state that corresponds to a $p$-like-state electron distribution. We therefore see that the rate-limiting step in the solvation dynamics of the electron is really an energyrelaxation process, referred to as a radiationless transition in molecules, that is consistent with the nonadiabatic process hypothesized by Rossky and Schnitker. ${ }^{29}$ One anticipates that the internal conversion process can be described by the terms that were neglected in the Born-Oppenheimer approximation, as is the case with molecules. For the solvated electron, it is reasonable to say that the relevant motions may be water vibrations and translations.

We have examined the solvation dynamics of an electron in $\mathrm{D}_{2} \mathrm{O}$. We found that the solvation dynamics are approximately $30 \%$ slower in $\mathrm{D}_{2} \mathrm{O}$ than in $\mathrm{H}_{2} \mathrm{O}$. Although this result is consistent with the prediction of the DCM because of an increase in the dielectric-relaxation time, ${ }^{53}$ the observed two-state dynamics and the hypothesis that the wet electron is an excited state of the solvated electron is fundamentally inconsistent with the DCM. The difference in the solvation dynamics could be due to the lowering of intermolecular and intramolecular vibrations and rota- 


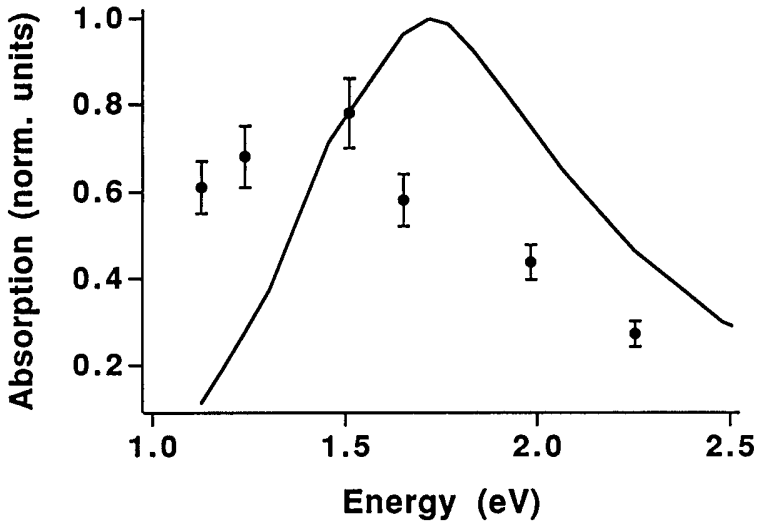

Fig. 8. Absorption of wet electron (filled circles) and solvated electron (curve). The error bars are \pm 1 standard deviation. Note the large component of the wet-electron absorption in the visible and the broad maxima near $1.4 \mathrm{eV}$.

tions on isotope substitution. Therefore any internal conversion process would be slowed. In contrast, we note that recent work of Barbara and his colleagues with fluorescence upconversion has shown that the DCM qualitatively predicts the dynamics of solvation in water and alcohols. $^{54,55}$

\section{Geminate Recombination}

Typical data for pulse radiolysis of water at three different probe wavelengths is plotted in Fig. 9. All studied wavelengths show the same kinetic behavior. ${ }^{10,11}$ Since there is no clear wavelength dependence of the data, the results suggest strongly that a single species or process is being observed. Except for the solvated electron, all species that are known to be produced by pulse radiolysis of water absorb weakly in the UV and have no absorption in the visible. Therefore we can be confident that we are looking only at the solvated electron. With respect to the possibility of the solvated electron's undergoing a quenching reaction, it is well established that reactions of the solvated electron with good quenchers, such as $\mathrm{O}_{2}$, are not important on the picosecond time scale. ${ }^{46}$ In addition, it is generally believed that the water cation is unstable in liquid water and within a few tens of femtoseconds reacts with neutral water to form $\mathrm{H}_{3} \mathrm{O}^{+}$and $\mathrm{OH}$ (Ref. 56):

$$
\begin{aligned}
& \mathrm{H}_{2} \mathrm{O}^{+}+\mathrm{H}_{2} \mathrm{O} \rightarrow \mathrm{OH}^{-}+\mathrm{H}_{3} \mathrm{O}^{+}, \\
& e_{\mathrm{aq}}{ }^{-}+\mathrm{H}_{3} \mathrm{O}^{+} \rightarrow \mathrm{H}_{2} \mathrm{O}+\frac{1}{2} \mathrm{H}_{2}, \\
& e_{\mathrm{aq}}{ }^{-}+\mathrm{OH} \rightarrow \mathrm{OH}^{-} .
\end{aligned}
$$

Thus we are actually observing the recombination of the solvated electron and the $\mathrm{H}_{3} \mathrm{O}^{+}$. The solvated electron can also react with the $\mathrm{OH}$ radical; however, since there is a Coulombic attraction between the solvated electron and the cation at short times, the dominant reaction is the electron's recombining with the cation.

We have also examined the recombination dynamics of solvated electrons in $\mathrm{D}_{2} \mathrm{O}$. A clear isotope effect is observed. Electron-pulse radiolysis experiments have seen isotope effects on the nanosecond time $\mathrm{scal}^{57}$; however, the interpretation of these measurements is quite difficult at best. The possibility of nongeminate recombination and complex spur dynamics cannot be neglected. From the solvated-electron geminate recombination kinetics in $\mathrm{D}_{2} \mathrm{O}$, we find that the recombination is slower and more electrons appear to escape recombination. If more electrons escape recombination, then the electron thermalization distance must be larger in $\mathrm{D}_{2} \mathrm{O}$. Transport properties, such as diffusion coefficients and viscosity, determine only the time-dependent kinetics of the system, whereas the escape yield given by the Onsager result is dependent only on the initial electron-cation separation distance. To estimate the solvated-electron thermalization distance carefully and to verify the existence of an isotope dependence on the electron thermalization distance, we have compared our data with values predicted by the Onsager model of electron-cation recombination. Results of the fitting are shown in Fig. 9. The agreement of the solvated-electron geminate recombination data in $\mathrm{D}_{2} \mathrm{O}$ with the Onsager model is quite good. With an exponential distribution of solvated electrons, a thermalization length of $12.4 \pm 0.3 \AA$ is obtained. The error in the estimate of the thermalization distance in water is much less than for the alkanes because of the much smaller escape yield. Similar estimates $(\sim 15 \AA)$ for the electron thermalization length can be obtained by several simpler methods as well. ${ }^{10,11}$ Measurements by more indirect methods and calculations support the idea of a short electron thermalization length in water..$^{58,59}$ The comparison of the Onsager model with solvated-electron geminate recombination in $\mathrm{H}_{2} \mathrm{O}$ was not as good. The approximate thermalization distance in $\mathrm{H}_{2} \mathrm{O}$ is $11.5 \pm 0.5 \AA$. Estimates from the Onsager model indicate that the isotope effect results in a larger thermalization length for $D_{2} \mathrm{O}$ than in $\mathrm{H}_{2} \mathrm{O}$. We believe that the physical basis for a larger ther-

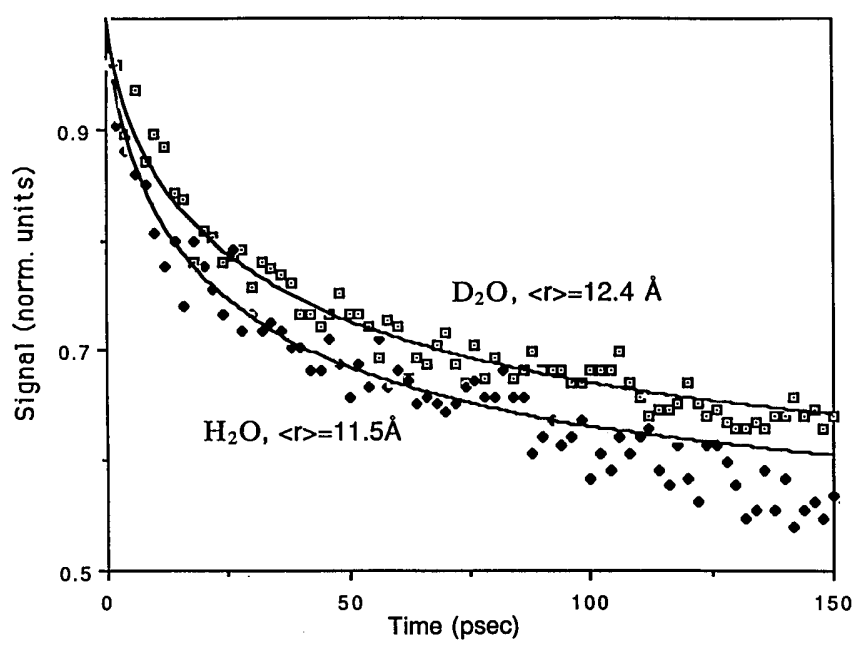

Fig. 9. Geminate recombination of solvated electron in $\mathrm{H}_{2} \mathrm{O}$ and $\mathrm{D}_{2} \mathrm{O}$, superimposed with fits to the Onsager model of geminate electron-cation recombination. $D=13.5 \times 10^{-5} \mathrm{~cm}^{2} / \mathrm{sec}$ in $\mathrm{H}_{2} \mathrm{O}$; for $\mathrm{D}_{2} \mathrm{O}$ the diffusion coefficient was assumed to scale with viscosity. Thermalization lengths of $12.4 \AA$ for $\mathrm{D}_{2} \mathrm{O}$ and $11.5 \AA$ for $\mathrm{H}_{2} \mathrm{O}$ were found. Possible explanations for the deviation between the Onsager model and the recombination in water are discussed in the text. 
malization length for $\mathrm{D}_{2} \mathrm{O}$ than $\mathrm{H}_{2} \mathrm{O}$ is a less efficient energy transfer from the hot photoionized electron to $\mathrm{D}_{2} \mathrm{O}$ than to $\mathrm{H}_{2} \mathrm{O}$. Thus more collision-transfer steps are needed to thermalize the hot electron in $\mathrm{D}_{2} \mathrm{O}$ than in $\mathrm{H}_{2} \mathrm{O}$, so a larger thermalization distance results.

\section{Electrons Photodetached from Simple Ions in Water}

For many years it has been known that many simple ions, e.g., $\mathrm{OH}^{-}$and $\mathrm{Cl}^{-}$, have strong, broad, featureless absorptions in the UV. It was first noticed more than 30 years ago that, when these ions absorbed light, solvated electrons were produced..$^{60,61}$ Originally it was thought that photoionization was occurring; however, this was later shown to be an incomplete explanation. This transition is called a charge transfer to the solvent spectrum. ${ }^{62-64}$ The generally accepted picture is the following. On absorbing light, the solvated ion goes into an excited state in which the electron is spread over the $\mathrm{H}_{2} \mathrm{O}$ molecules that surround the ion. Experimentally it is found that a large fraction of these electrons then escape over the barrier and become solvated electrons. In this paper we report on the solvation dynamics and geminate recombination dynamics of electrons that are produced by charge transfers to the solvent spectrums.

Figure 10 shows the appearance of the solvated electron in neat water and a $1 \mathrm{M}$ solution of $\mathrm{NaCl}$. The electron that originates from the $\mathrm{Cl}^{-}$solvates significantly slower than the electron from the $\mathrm{H}_{2} \mathrm{O}$. When the signal from $n$-octane is used as an instrumental response, a rise time of $500 \mathrm{fsec}$ is found for the $\mathrm{Cl}^{-}$, compared with $350 \mathrm{fsec}$ for the $\mathrm{H}_{2} \mathrm{O}$. The solvation time for the electron originating from the $\mathrm{OH}^{-}$was also found to differ from that from neat water. These results are inconsistent with continuum theories of electron solvation, which predict that there will be almost no change in the observed solvation dynamics. ${ }^{65}$ We thus conclude that molecular effects are playing a large

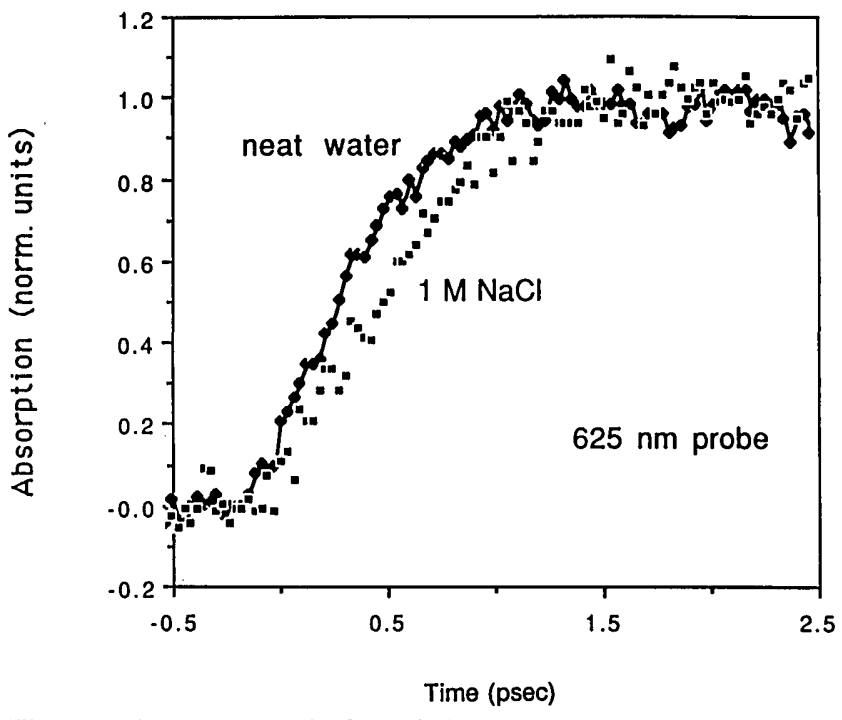

Fig. 10. Appearance of solvated electron in water following ejection from a chloride ion (filled squares) and from a neutral water molecule (diamonds). The line that connects the neat-water data was drawn to aid the viewer. The signals occur with average rise times of 350 and 500 fsec, respectively. Both experiments were done at room temperature with a 625 -nm probe.

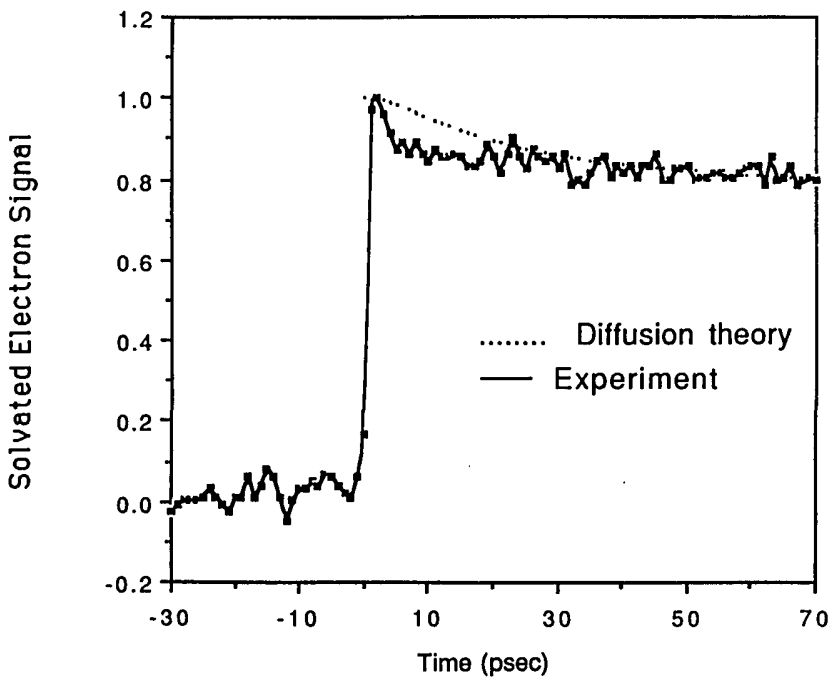

Fig. 11. Decay of solvated electron that originates from a Cl${ }^{-}$ion. Superimposed is a plot (dotted curve) of the diffusive recombination of hard spheres, with the following parameters: The mutual diffusion coefficient $D=0.7 \times 10^{-4} \mathrm{~cm}^{2} / \mathrm{sec}$; the initial separation of the two species, with respect to their centers, $R=8.5 \AA$; the reaction radius $r_{a}=3 \AA$.

role in the electron-solvation dynamics. If the electron that is ejected from the state of charge transfer to the solvent spectrum is near the atom, then the solvent structure around the previously solvated species can greatly influence the solvation dynamics. As discussed in our previous paper, in our multiphotonionization experiments the electron thermalization distance is shown to be $\sim 15 \AA .{ }^{10,11}$ At these larger electron-atom separations, much smaller local solvent effects would be expected.

At longer times, decays are seen in the solvated-electron absorption signal (Fig. 11). The decays seen in $\mathrm{NaCl}$, $\mathrm{NaOH}$, and neat water are all different from one another. ${ }^{11,12}$ It is known that solvated electrons do not react with $\mathrm{Na}^{+}, \mathrm{Cl}^{-}$, or $\mathrm{OH}^{-}$ions on the time scale of these experiments. ${ }^{4}$ With the additional fact that no concentration dependence was seen in the decay, the possibility that the solvated-electron reacts with the $\mathrm{Na}^{+}$or the $\mathrm{Cl}^{-}$ions is discounted; concentrations of as much as $1 \mu \mathrm{M}$ were studied. Therefore we conclude that the observed decays are due to geminate recombination of the electron with the $\mathrm{Cl}$ atom or the $\mathrm{OH}$ radical that is formed on photodetachment of the electron by the UV pulse. The marked differences that we observed between the neat water and the ionic solution experiments are thus expected, since in the former the electron recombines with an ion, whereas in the latter the electron recombines with a neutral.

We have compared our data with values predicted by a continuum diffusion model of neutral-pair recombination; see Subsection 3.B. We see from Fig. 11 that the best fit that we can obtain by using Eq. (9) is poor. The fit was not significantly improved by using a distribution. Although we have not completed a quantitative comparison, we find that the molecular theory of Northrup and Hynes yields a trend that is consistent with our results. ${ }^{66}$ That is, at short times a larger amount of recombination takes place than would be predicted by continuum theories. It 
should, however, be noted that there will be a force of attraction between the electron and the atom because of the polarizibility of the $\mathrm{Cl}$ atom. At the earliest times, i.e., before the water molecules rearrange around the nascent $\mathrm{Cl}$ atom, there is a strong attractive force between the electron and neutral hole because of polarization forces. We are at present investing these possibilities because they may be the origin of the observed fast recombination.

\section{SUMMARY}

We have used femtosecond spectroscopy to study electrons in a variety of liquid environments. Geminate electroncation recombination has been observed in polar and nonpolar liquids. The observed recombination kinetics in neat alkanes are found to agree well with the Onsager model of ion-pair recombination. This model includes the assumption that the motion of the electron can be treated classically as a Brownian particle in a Coulombic force field. Our results suggest that the quantum-mechanical nature of the electron is important in determining the electron's spectral and transport properties but is not vital in the description of its motion on the time scale of the electron-cation recombination.

The recombination of solvated electrons in water is more complex. Good agreement was found between the Onsager model and the recombination data in $\mathrm{D}_{2} \mathrm{O}$; however, the agreement between the model and the recombination in $\mathrm{H}_{2} \mathrm{O}$ was not satisfactory. Estimates of the electron thermalization distance that use the Onsager model indicate that it is quite short, $12.4 \pm 0.3 \AA$ for $\mathrm{D}_{2} \mathrm{O}$ and $11.5 \pm 0.5 \AA$ for $\mathrm{H}_{2} \mathrm{O}$. Our experiments indicate that there is an isotope dependence for the electron thermalization length. The larger thermalization length found in $\mathrm{D}_{2} \mathrm{O}$ is attributed to an energy-transfer effect. Whether a continuum description of the solvent is truly appropriate for the description of geminate recombination kinetics at such small distances is not known. Certainly the finite sizes of the solvated electron and the hydronium cation must be considered in more detail. An improved model would account for the known reaction of the solvated electron and the $\mathrm{OH}$ radical in addition to finite size effects. Further work is planned in this direction.

Electron-solvation dynamics were studied in neat water and in aqueous solution. The formation, decay, and absorption spectra of the wet electron in neat water were measured. By the discovery of an isosbestic wavelength, the formation of the solvated electron from the wet electron was shown to involve only two states; i.e., the transition from the wet electron to the solvated electron is not continuous. This is fundamentally inconsistent with the dielectric relaxation model of solvation dynamics. The picture of the wet electron as an excited state of the solvated electron was presented. Therefore the ratelimiting step in the electron-solvation dynamics is an internal conversion, which is consistent with the nonadiabatic process proposed by Rossky and Schnitker. ${ }^{29}$ In addition, an isotopic dependence of the electron-solvation dynamics has been found in neat water and in $\mathrm{D}_{2} \mathrm{O}$.

Studies of photodetachment from simple ions in water have shown that the electron that originates from a neutral $\mathrm{H}_{2} \mathrm{O}$ molecule and that from a simple ion such as $\mathrm{Cl}^{-}$ have distinctly different solvation dynamics. This is not predicted by the DCM of solvation. A fraction of the electrons that are photodetached from the ion after solvation is observed to recombine with the parent atom. The observed kinetics are not consistent with continuum models of recombination. We believe that these deviations are due to short-range molecular effects.

\section{ACKNOWLEDGMENTS}

We thank R. Bowman for his early contributions to this work and Xuelong Shi for his assistance with the data analysis. Furthermore, we thank the National Science Foundation, the U.S. Air Force Office of Scientific Research, and the Petroleum Fund administered by the American Chemical Society for their generous financial support. F. H. Long thanks IBM for a graduate student fellowship.

\section{REFERENCES AND NOTES}

1. W. Weyl, Pogg. Ann. 123, 350 (1864).

2. E. Rutherford, Radioactivity 33 (1904).

3. E. J. Hart and J. W. Boag, "Absorption spectra of the hydrated electron in water and aqueous solutions," J. Am. Chem. Soc. 84, 4090 (1962).

4. A. K. Pikaev, The Solvated Electron in Radiation Chemistry (World University, Jerusalem, 1970).

5. Farhataziz and M. A. J. Rodgers, eds., Radiation Chemistry Principles and Applications (VCH, New York, 1987).

6. G. R. Freeman, ed., Kinetics of Inhomogeneous Processes (Wiley, New York, 1987).

7. N. F. Mott and E. A. Davis, Electronic Processes in NonCrystalline Materials, 2nd. ed. (Oxford U. Press, New York, 1979).

8. R. A. Marcus and N. Sutin, "Electron transfers in chemistry and biology," Biochim. Biophys. Acta 811, 265 (1985).

9. R. M. Bowman, H. Lu, and K. B. Eisenthal, "Femtosecond study of electron-hole recombination in neat alkanes," J. Chem. Phys. 89, 606 (1988).

10. H. Lu, F. H. Long, R. M. Bowman, and K. B. Eisenthal, "Femtosecond studies of electron-cation recombination in water," J. Phys. Chem. 93, 27 (1989).

11. F. H. Long, H. Lu, and K. B. Eisenthal, "Femtosecond studies of electron-cation dynamics in water: the role of isotope substitution," Chem. Phys. Lett. 160, 464 (1989).

12. F. H. Long, H. Lu, and K. B. Eisenthal, "Femtosecond studies of electron photodetachment from simple ions in liquid water: solvation and geminate recombination dynamics," J. Chem. Phys. 91, 4193 (1989).

13. T. W. Scott and C. L. Braun, "Picosecond measurements of geminate charge pair recombination in photoionized liquids," Can. J. Chem. 63, 228 (1985); "Picosecond geminate charge recombination: the dependence of reaction kinetics on initial pair recombination," Chem. Phys. Lett. 127, 501 (1986); C. L. Braun and T. W. Scott, "Picosecond absorption measurements of geminate electron-cation recombination," J. Phys. Chem. 91, 4436 (1987).

14. F. H. Long, H. Lu, and K. B. Eisenthal, "Femosecond studies of the pre-solvated electron: an excited state of the solvated electron?" Phys. Rev. Lett. 64, 1469-1472 (1990).

15. H. Frohlich, Theory of Dielectrics (Oxford U. Press, New York, 1949).

16. Y. T. Mazurenko and N. G. Bakshiev, "Effects of orientation dipole relaxation on spectral time, and polarization characteristics of the luminescence of solutions," Opt. Spectrosc. (USSR) 28, 490 (1970).

17. B. Bagchi, D. W. Oxtoby, and G. R. Fleming, "Theory of timedependent Stokes-shift in polar media," Chem. Phys. 86, 257 (1984). 
18. B. J. Berne, "A self-consistant theory of rotational diffusion," J. Chem. Phys. 62, 1154 (1975).

19. D. F. Calef and P. G. Wolynes, "Smoluchowski-Vlasov theory of charge solvation," J. Chem. Phys. 78, 4145 (1983).

20. G. van der Zwan and J. T. Hynes, "Time-dependent flourescence solvent shifts, dielectric friction and nonequilibrium solvation in polar liquids," J. Phys. Chem. 89, 4181 (1985).

21. H. Sumi and R. Marcus, "Dielectric relaxation and intermolecular electron transfers," J. Chem. Phys. 85, 4272 (1986).

22. R. F. Loring and S. Mukamel, "Molecular theory of solvation and dielectric response in polar liquids," J. Chem. Phys. 87, 1272 (1987).

23. V. Friedrich and D. Kivelson, "Theory of time-dependent polarization about an ion in an isotropic fluid," J. Chem. Phys. 86, 6425 (1987).

24. P. Wolynes, "Linearized microscopic theories of nonequilibrium charge solvation," J. Chem. Phys. 86, 5133 (1987).

25. I. Rips, J. Klafter, and J. Jortner, "Solvation dynamics in polar liquids," J. Chem. Phys. 89, 4288 (1988).

26. A. L. Nichols and D. F. Calef, "Polar solvent relaxation in the mean spherical approximation approach," J. Chem. Phys. 89, 3783 (1988).

27. J. Schnitker, K. Motakabbir, P. J. Rossky, and R. Friesner, "A priori calculation of the optical absorption spectra of the hydrated electron," Phys. Rev. Lett. 60, 456 (1988).

28. A Wallqvist, G. Martyna, and B. J. Berne, "Behavior of the solvated electron at different temperatures: structure and absorption spectrum." J. Phys. Chem. 92, 1721 (1988).

29. P. J. Rossky and J. Schnitker, "The hydrated electron: quantum simulation of structure, spectroscopy, and dynamics," J. Phys. Chem. 92, 4277 (1988).

30. R. B. Barnett, U. Landman, and A. Nitzan, "Relaxation dynamics following transitions of solvated electron," J. Chem. Phys. 90, 4413 (1989).

31. L. Onsager, "Initial recombination of ions," Phys. Rev. 54, 554 (1938).

32. M. Tachiya, "General method for calculating the ecape probability in diffusion controlled reactions," J. Chem. Phys. 69, 2375 (1978).

33. A Mozumder, "Theory of neutralization of an isolated ion pair: application of the method of prescribed diffusion to random walk in a Coulomb field." J. Chem. Phys. 48, 16591665 (1968).

34. N. J. B. Green, M. J. Pilling, S. M. Pimblott, and P. Clifford, "Stochastic models of diffusion-controlled ionic reactions in radiation-induced spurs. 2 Low permittivity solvents," J. Phys. Chem. 93, 8025 (1989).

35. K. M. Hong and J. Noolandi, "Solution of the Smoluchowski equation with a Coulomb potential. I. General results," J. Chem. Phys. 68, 5163 (1978); "Solution of the Smoluchowski equation with a Coulombic potential. II. Application to flourescence quenching," J. Chem. Phys. 68, 5172 (1978); "Solution of the time-dependent Onsager problem," J. Chem. Phys. 68, 5026 (1978).

36. F. C. Collins and G. E. Kimball, "Diffusion controlled reaction rates," J. Colloid Sci. 4, 425 (1949).

37. J. Schnitker and P. J. Rossky, "Excess electron migration in liquid water," J. Phys. Chem. 94, 6965 (1989).

38. H. L. Friedman, "A hydrodynamic effect in the rates of diffusion controlled reactions," J. Phys. Chem. 70, 3931 (1966).

39. M. G. Robinson and G. R. Freeman, "Electron mobilities and ranges in liquid C1-C3 hydrocarbons and in xenon: effect of temperature and field strength," Can. J. Chem. 52, 440 (1971).

40. W. F. Schmidt, "Electron mobility in nonpolar liquids; the effect of molecular structure, temperature and electric field," Can. J. Chem. 55, 2197 (1977).

41. W. F. Schmidt and A. O. Allen, "Free-ion yield in sundry irradiated liquids," J. Chem. Phys. 52, 5 (1970).

42. Y. Yoshida, S. Tagawa, H. Kobayashi, and Y. Tabata, "Study of geminate ion recombination in a solute-solvent system by using picosecond pulse radiolysis," Radiat. Phys. Chem. 30, 83 (1987); "Study of geminate ion recombination non-polar liquids," Radiat. Phys, Chem. 28, 201 (1986); S. Tagawa, M. Washio, H. Kobayashi, Y. Katsumura, and Y. Tabata, "Pi- cosecond pulse radiolysis studies on geminate ion recombination in saturated hydrocarbon," Radiat. Phys. Chem. 21, 45 (1983).

43. C. D. Jonah, "Decay of geminate ions in hexanes," Radiat. Phys. Chem. 21, 53 (1983).

44. A simple integration of $r g(r)$ over all space shows that $\langle r\rangle=$ $3 L$. Furthermore, care must be taken not to confuse $g(r)$, the probability density, with $4 \pi r^{2} g(r)$, the probability of the electron's being at a distance $r$ away from the origin.

45. W. P. Helman, "Quenching of alkane fluorescence by tetrachloromethane measured by nanosecond decay time techniques," Chem. Phys. Lett. 17, 2, 306 (1972).

46. W. J. Chase and J. W. Hunt, "Solvation time of the electron in polar liquids: water and alcohols," J. Phys. Chem. 79, 2835 (1975).

47. Y. Wang, M. K. Crawford, M. J. McAuliffe, and K. B. Eisenthal, "Picosecond laser studies of electron solvation in alcohols," Chem. Phys. Lett. 74, 160 (1980).

48. G. A. Kenney-Wallace and C. D. Jonah, "Picosecond spectroscopy and solvation clusters. The dynamics of localizing electrons in polar fluids," J. Phys. Chem. 86, 2572 (1982).

49. J. M. Wiesenfeld and E. P. Ippen, "Dynamics of electron solvation in liquid water," Chem. Phys. Lett. 73, 47 (1980).

50. A Migus, Y. Gaudel, J. L. Martin, and A. Antonetti, "Femtosecond studies of electrons in water: first evidence of the pre-hydrated state," Phys. Rev. Lett. 58, 1559 (1987).

51. B. D. Micheal, E. J. Hart, and K. H. Schmidt, "The solvated electron absorption at $25^{\circ} \mathrm{C}$," J. Phys. Chem. 75, 2798 (1971).

52. J. Schnitker, P. J. Rossky, and G. A. Kenney-Wallace, "Electron localization in liquid water: a computer simulation study of microscopic trapping sites," J. Chem. Phys. 85, 2989 (1986).

53. D. Eisenberg and W. Kauzmann, The Structure and Properties of Water (Oxford U. Press, New York, 1969).

54. M. Maroncelli and G. R. Flemming, "Picosecond solvation dynamics of Coumarin 153: the importance of molecular aspects of solvation," J. Chem. Phys. 86, 6221 (1987).

55. W. Jarzeba, G. C. Walker, A. E. Johnson, and P. F. Barbara, "Femtosecond microscopic solvation dynamics of aqueous electrons," J. Phys. Chem. 92, 7039 (1988).

56. F. W. Lampe, F. H. Field, and J. L. Franklin, "Reactions of gaseous ions IV. Water," J. Am. Chem. Soc. 79, 6132 (1957).

57. A C. Chernovitz and C. D. Jonah, "Isotopic dependence of recombination kinetics in water," J. Phys. Chem. 92, 5946 (1988).

58. V. V. Konovalov, A. Raitsimring, Yu. D. Tsvetkov, and V. A. Benderskii, "The thermalization length of low-energy electrons determined by nanosecond photoemission into aqueous electrolyte solution," Chem. Phys. 93, 163 (1985).

59. T. Goulet and J. P. Jay-Gerin, "Thermalization distances and times for electrons in water," J. Phys. Chem. 92, 6871 (1988).

60. L. I. Grossweiner and M. S. Matheson, "The kinetics of the dihalide ions from the flash photolysis of aqueous halide solutions," J. Phys. Chem. 61, 1089 (1957).

61. M. S. Matheson, W. A. Mulac, and J. Rabani, "Formation of the hydrated electron in the flash photolysis of aqueous solutions," J. Phys. Chem. 67, 261 (1963).

62. For a general review see M. J. Blandamer and M. F. Fox, "Theory and applications of charge-transfer-to-solventspecta," Chem. Rev. 70, 59 (1970) and the references therein.

63. J. Jortner, M. Ottolenghi, and G. Stein, "Cage effects and scavenging mechanisms in the photochemistry of the iodide ion in aqueous solution," J. Phys. Chem. 66, 2029 (1962); "The effect of nitrous oxide and the nature of intermediates in the photochemistry of the iodide ion in solution," J. Phys. Chem. 66, 2037 (1962).

64. F. S. Dainton and S. R. Logan, "Primary processes in the photolysis of the iodide ion in aqueous solution," Proc. R. Soc. London Ser. A 287, 281 (1965).

65. From the known dielectric properties of a $1 \mathrm{M} \mathrm{NaCl}$ solution, a $7 \%$ increase in the longitudinal response time is expected.

66. S. Northrup and J. T. Hynes, "Short range caging effects for reaction in solution. II. Escape probabilities and time dependent reactivity," J. Chem. Phys. 71, 884 (1979). 